\title{
PENETRATION DEPTH OF CLOSELY SPACED WATER-FREE CREVASSES
}

\author{
By J. Weertman \\ (Department of Materials Science and Engineering and Department of Geological Sciences, \\ Northwestern University, Evanston, Illinois 6o20 I, U.S.A.)
}

Abstract. An approximate, analytic solution is found for the profile of a water-free crevasse in a field
of closely spaced crevasses. The depth of penetration of the crevasses into the glacier is found. If the fracture
strength of ice is taken to be zero, the penetration depth is equal to the value found by Nye and is independent
of the crevasse spacing. This conclusion is in disagreement with results reported recently by R. A. Smith.
If the fracture strength of ice is taken to be finite, the penetration depth is reduced if the spacing between
crevasses is reduced.
The results of the analysis can be applied to other crack problems. In particular, it can be applied to
thermal, secondary cracking that it is hoped occurs when cooling fluid flows through the cracks created by
hydraulic fracture for the purpose of extracting geothermal heat from hot, dry rock masses.

RÉsumé. Profondeur de pénétration des crevasses libres d'eau en reseau dense. On donne une solution analytique du profil d'une crevasse dépourvue d'eau liquide dans un champ de crevasses rapprochées. On trouve la profondeur de pénétration de la crevasse dans le glacier. Si la limite de rupture de la glace est prise égale à zéro, la profondeur de pénétration est égale à la valeur trouvée par Nye et indépendante de l'espacement entre les crevasses. Cette conclusion est en désaccord avec les résultats récemment rapportés par R. A. Smith. Si la limite de rupture de la glace est prise à une valeur finie, la profondeur de pénétration est réduite lorsque l'espacement entre les crevasses est réduit.

Les résultats de l'analyse peuvent être appliqués à d'autres problèmes de fissuration. En particulier, on peut l'appliquer aux fissurations thermiques secondaires que l'on pense devoir se produire lorsqu'un fluide froid s'écoule à travers des fissures créées par rupture hydraulique dans le but d'extraire la chaleur géothermique à partir de masses rocheuses sèches et chaudes.

Zusammenfassung. Die Eindringtiefe eng benachbarter, wasserfreier Spalten. Das Profil einer wasserfreien Spalte in einem Feld eng benachbarter Spalten lässt sich angenähert berechnen. Daraus ergibt sich die Eindringtiefe der Spalten in das Gletschereis. Wenn die Bruchfestigkeit von Eis gleich Null gesetzt wird, ist die Eindringtiefe gleich dem von Nye gefundenen Wert und unabhängig vom Spaltenabstand. Dieser Schluss steht im Widerspruch zu Ergebnissen, die unlängst von R. A. Smith mitgeteilt wurden. Wenn die Bruchfestigkeit des Eises ungleich Null gesetzt wird, nimmt die Eindringtiefe mit verringertem Spaltenabstand $\mathrm{ab}$.

Die Ergebnisse der Analyse lassen sich auf andere Bruchprobleme anwenden. Ein spezielles Anwendungsgebiet liegt in der thermischen, sekundären Rissbildung, die - so hofft man — dann eintritt, wenn Kühlfüssigkeit durch Risse dringt, die durch hydraulischen Bruch zum Zwecke der Gewinnung der Erdwärme aus heissen, trockenen Felsmassen erzeugt wurden.

\section{INTRODUCTION}

One conclusion reached by Smith (1976) in his recent paper is that even if the fracture strength of ice is zero, the depth of penetration of water-free crevasses into a glacier is reduced if the spacing between the crevasses is reduced. He found that in the limit in which the spacing goes to zero, the penetration depth also goes to zero. Previously (Nye, r 955; Robin, 1974; Weertman, 1973, 1974) it had been concluded that the penetration depth of closely spaced, water-free crevasses was equal to the Nye depth of $\mathcal{T} / \rho g$ where $\rho$ is the density of ice, $g$ is the gravitational acceleration and $T$ is the tensile stress acting within the glacier. This depth is independent of the spacing.

None of the papers that considered the question of the penetration depth of closely spaced, water-free crevasses actually presented the analytical solution (nor even a numerical solution) of the profile of a crevasse in a field of closely spaced crevasses. The solution of this problem obviously could resolve the controversy between Smith's work and the older work on the question of penetration depth. In this paper I wish to present an analytical solution of the crevasse profile. This solution supports the older conclusion that the penetration depth of closely spaced, water-free crevasses is equal to $T / \rho g$ and is independent of the crevasse spacing. Only if ice has a finite fracture strength can the crevasse penetration depth be reduced by reducing the crevasse spacing. I solve first the problem of parallel, closely spaced cracks in an 
infinite elastic medium. It is then shown that the solution to this problem is essentially the same as the solution to the problem of closely spaced crevasses. The latter problem is one of closely spaced, parallel edge cracks in an elastic half-space.

\section{ANALysis}

Consider an infinite set of vertical, equally spaced, parallel cracks in an infinite solid. Let the spacing between the cracks be equal to $h$ and let the centers of the cracks be situated at $y=0$ and $x=0, \pm h, \pm 2 h, \pm 3 h, \ldots$. The cracks extend in the vertical $y$ direction between $-a \leqslant y \leqslant a$. Because the cracks will be considered to be closely spaced the ratio $h / a \ll \mathrm{I}$.

It is assumed that before the cracks were introduced into the solid the stress $\sigma_{x x}(y)$ was given by

$$
\sigma_{x x}(y)=T-\rho g|y|
$$

It is further assumed that $\sigma_{y y}(y)$ is a function only of $y$ and that the other stress components are zero with the exception of $\sigma_{z z}(y)$, which is equal to $\nu\left(\sigma_{x x}+\sigma_{y y}\right)$ where $\nu$ is Poisson's ratio.

After the cracks are introduced into the solid, the normal and shear stresses across the crack surfaces, that is the surface tractions, are set equal to zero. Let $D(y)$ represent the crack opening displacement across any one of the cracks and let $B(y)=-\mathrm{d} D(y) / \mathrm{d} y$. (A crack can be considered to be a collection of infinitesimal dislocations. If it is, then $B(y)$ represents the density of these dislocations.)

In order for the crack surfaces to be traction-free the following fundamental equation that applies to the problem of an infinite set of parallel cracks must be satisfied in the region $-a \leqslant y \leqslant a$ :

where

$$
[\mu / 2 \pi(\mathrm{I}-\nu)] \int_{-a}^{a} B\left(y^{\prime}\right) G\left(y-y^{\prime}\right) \mathrm{d} y^{\prime}=-\sigma_{x x}(y)=-\mathcal{T}+\rho g|y|,
$$

$$
\begin{aligned}
G\left(y-y^{\prime}\right)=(\pi / h)\left\{2 \sinh \left[\pi\left(y-y^{\prime}\right) / h\right] \cosh \left[\pi\left(y-y^{\prime}\right) / h\right]-\right. \\
\left.-\left[\pi\left(y-y^{\prime}\right) / h\right]\right\} / \sinh ^{2}\left[\pi\left(y-y^{\prime}\right) / h\right],
\end{aligned}
$$

and $\mu$ is the shear modulus. Equation (2) was derived by Smith (1966[a], [b]) and by Yokobori and co-workers (Ichikawa and others, I965; Yokobori and Ichikawa, I967[a], [b]). It is valid for all values of the ratio $h / a$. Equation (2) determines the crack profile $D(y)$.

An approximate solution of Equation (2) now will be constructed for the case of interest, that is, when $h / a \ll \mathrm{I}$. The solution of the crack profile is determined separately in three regions: for values of $y$ within the limits $h<|y|<(a-h)$, within the limits $-h<y<h$, and within the limits $(a-h)<|y|<a$.

The region $h<|y|<(a-h)$

Note that when the ratio $\left|y-y^{\prime}\right| / h$ is large, the function $G \approx 2 \pi / h$ when $y>y^{\prime}$ and $G \approx-2 \pi / h$ when $y<y^{\prime}$. Thus when $y$ has a value such that both of the terms $|y|$ and $(a-|y|)$ are large compared with the spacing $h$, the integral that appears in Equation (2) is given by

$$
\begin{aligned}
I(y) & \equiv \int_{-a}^{a} B\left(y^{\prime}\right) G\left(y-y^{\prime}\right) \mathrm{d} y^{\prime} \\
& \approx \int_{-a}^{-a+h}(2 \pi / h) B\left(y^{\prime}\right) \mathrm{d} y^{\prime}+\int_{-a+h}^{-h} B\left(y^{\prime}\right) G\left(y-y^{\prime}\right) \mathrm{d} y^{\prime} \pm \int_{-h}^{h}(2 \pi / h) B\left(y^{\prime}\right) \mathrm{d} y^{\prime}+
\end{aligned}
$$




$$
\begin{gathered}
+\int_{h}^{a-h} B\left(y^{\prime}\right) G\left(y-y^{\prime}\right) \mathrm{d} y^{\prime}+\int_{a-h}^{a}(-2 \pi / h) B\left(y^{\prime}\right) \mathrm{d} y^{\prime} \\
=-(4 \pi / h) D(a-h)+\int_{-a+h}^{-h} B\left(y^{\prime}\right) G\left(y-y^{\prime}\right) \mathrm{d} y^{\prime}+\int_{h}^{a-h} B\left(y^{\prime}\right) G\left(y-y^{\prime}\right) \mathrm{d} y^{\prime},
\end{gathered}
$$

where use has been made of the symmetry relationship $D(y)=D(-y)$ and $D(a)=$ $D(-a)=0$. The plus sign is used in Equation (4) if $y>0$ and the minus sign if $y<0$. Since $\int_{-h}^{h} B\left(y^{\prime}\right) \mathrm{d} y^{\prime}=0$ it makes no difference which sign is used.

Assume next that the displacement $D(y)$ is given approximately by

$$
D(y)=\alpha(T-\rho g|y|),
$$

where $\alpha$ is a constant. (The reason for choosing this function is obvious. If a long thin strip of material were subjected to a normal stress equal to $T-\rho g|y|$ the surfaces of the strip would be displaced by an amount approximately proportional to $T-\rho g|y|$.) If Equation (5) is used to determine $B(y)$ and $B(y)$ is then inserted into Equation (4) and use is made of the standard integrals

$$
\int(\cosh x / \sinh x) \mathrm{d} x=\log |\sinh x|
$$

and

$$
\int\left(x / \sinh ^{2} x\right) \mathrm{d} x=-x \operatorname{coth} x+\log |\sinh x|
$$

the integral $I(y)$ of Equation (4) becomes

$$
\begin{aligned}
I(y)= & -(4 \pi / h) D(a-h)+(\alpha \rho g)(\pi / h)\{(y+h) \operatorname{coth}[\pi(y+h) / h]+ \\
& +(y-h) \operatorname{coth}[\pi(y-h) / h]-(y+a-h) \operatorname{coth}[\pi(y+a-h) / h]- \\
& -(y-a+h) \operatorname{coth}[\pi(y-a+h) / h]+ \\
& +(h / \pi) \log \mid\{\sinh [\pi(y+h) / h] \sinh [\pi(y-h) / h]\} /\{\sinh [\pi(y+a-h) / h] \times \\
& \times \sinh [\pi(y-a+h) / h]\}\} \\
\approx & (4 \pi / h)\{\alpha \rho g(|y|-a)-D(a-h)\} .
\end{aligned}
$$

If Equation (6) is inserted into Equation (2) it is seen that the constant $\alpha$ must be given by

$$
\alpha=h(\mathbf{I}-v) / 2 \mu,
$$

and the displacement $D(a-h)$ by

$$
D(a-h)=[h(\mathbf{I}-\nu) / 2 \mu][T-\rho g(a-h)] .
$$

The fact that the displacement $D(y)$ given by Equations (5) and (7) when inserted into Equations (2) and (4) produces a self-consistent solution justifies a posteriori the use of Equation (5) on mathematical as well as physical grounds.

The region $-h<y<h$

Consider next the region $-h<y<h$. Note that when $\left|y-y^{\prime}\right| / h$ is small the function $G$ is approximately equal to $1 /\left(y-y^{\prime}\right)$. Thus the integral $I(y)$ is approximately equal to 


$$
\begin{aligned}
I(y) & \approx \int_{-a}^{-h}(2 \pi / h) B\left(y^{\prime}\right) \mathrm{d} y^{\prime}+\int_{-h}^{h}\left(y-y^{\prime}\right)^{-1} B\left(y^{\prime}\right) \mathrm{d} y^{\prime}+\int_{h}^{a}(-2 \pi / h) B\left(y^{\prime}\right) \mathrm{d} y^{\prime} \\
& =-(4 \pi / h) D(h)+\int_{-h}^{h}\left(y-y^{\prime}\right)^{-1} B\left(y^{\prime}\right) \mathrm{d} y^{\prime} .
\end{aligned}
$$

If Equation (9) is inserted into Equation (2) the following equation is found

$$
\begin{aligned}
\int_{-h}^{h}\left(y-y^{\prime}\right)^{-1} B\left(y^{\prime}\right) \mathrm{d} y^{\prime} & =\{2 \pi(\mathrm{I}-\nu) / \mu\}\{-T+\rho g|y|\}+(4 \pi / h) D(h) \\
& \approx\{2 \pi(\mathrm{I}-v) / \mu\}\{\rho g(|y|-h\} .
\end{aligned}
$$

The solution of Equation ( 10 ) can be found using tables of Hilbert transforms. The solution for $D$ is

$$
D(y)=[h T(\mathrm{I}-v) / 2 \mu][\mathrm{I}+O(\rho g h / T)] \approx h T(\mathrm{I}-v) / 2 \mu .
$$

The region $(a-h)<|y|<a$

Finally, consider the crack tip regions $(a-h)<|y|<a$. The integral $I(y)$ in this region can be expressed as

$$
\begin{aligned}
I(y) & \approx \int_{-a}^{a-h}(2 \pi / h) B\left(y^{\prime}\right) \mathrm{d} y^{\prime}+\int_{a-h}^{a}\left(y-y^{\prime}\right)^{-1} B\left(y^{\prime}\right) \mathrm{d} y^{\prime} \\
& =-(2 \pi / h) D(a-h)+\int_{a-h}^{a}\left(y-y^{\prime}\right)^{-1} B\left(y^{\prime}\right) \mathrm{d} y^{\prime} .
\end{aligned}
$$

Substitution into Equation (2) produces

$$
\int_{a-h}^{a}\left(y-y^{\prime}\right)^{-1} B\left(y^{\prime}\right) \mathrm{d} y^{\prime} \approx[\pi(\mathbf{1}-v) / \mu][-T+2 \rho g|y|-\rho g a+\rho g h] .
$$

The solution of Equation (13) can be found with the aid of a table of Hilbert transforms and the condition that Equation (8) be satisfied. The solution is

$$
\begin{aligned}
& D(y) \approx[(\mathrm{I}-v) / \mu]\left\{(h / 4)[T-\rho g(a-h)-\pi \rho g h]\left[\mathrm{I}-(2 / \pi) \sin ^{-1}\left(y^{\star} / h^{\star}\right)\right]+\right. \\
& \left.\quad+\int_{y^{\star}}^{h^{\star}}\left\{[T-\rho g(a-h)]\left[y^{\star} /\left(h^{\star 2}-y^{\star 2}\right)^{\frac{1}{2}}\right]+(2 \rho g)\left(h^{\star 2}-y^{\star 2}\right)^{\frac{1}{2}}\right\} \mathrm{d} y^{\star}\right\},
\end{aligned}
$$

where $h^{\star}=h / 2, y^{\star}=|y|-a+h^{\star}$, and $y^{\star}$ is restricted to the range of values $-h^{\star}<y^{\star}<h^{\star}$.

The displacement $D(y)$ must always be a positive quantity. Therefore, the value of $a$ is restricted to the range

$$
0<a<T / \rho g \text {. }
$$

The tensile stress $T^{\star}$ across the crack plane ahead of, but very close to the crack tip is

$$
T^{\star}=K /(2 \pi r)^{\frac{1}{2}}
$$

where $r$ is distance from the crack tip and $K$ is a constant known as the stress intensity factor. The tensile stress becomes infinitely large as $r \rightarrow 0$. The stress intensity factor $K$ is equal to the limit

$$
K=-\left[\{\mu /(\mathrm{I}-\nu)\}(\pi / 2)^{\frac{1}{2}}(a-y)^{\frac{1}{2}}\{\mathrm{~d} D(y) / \mathrm{d} y\}\right]_{y \rightarrow a} .
$$


If Equation ( 14 ) is substituted into Equation ( 17 )

$$
K \approx(\pi h / 2)^{\frac{1}{2}}[(\pi+\mathrm{I}) / 2 \pi](T-\rho g a) .
$$

If the fracture strength of the solid is effectively zero, the cracks will propagate until the stress intensity factor $K$ becomes equal to zero. In other words, the crack half-length $a$ will increase until

$$
a=T / \rho g \text {. }
$$

This half-length is equal to the Nye depth of a crevasse. It should be noted that the halflength $a$ given by Equation (I9) is independent of the spacing $h$ between the cracks.

If the solid has a finite fracture strength the crack half-length will depend on $h$. Let $K_{\mathrm{c}}$ represent the critical $K$ value for crack propagation in the solid. Then according to Equation (I8) a crack will propagate until its half-length $a$ takes on the value

$$
a=(\rho g)^{-1}\left\{T-K_{\mathrm{c}}(2 \pi / h)^{\frac{1}{2}}[2 \pi /(\pi+\mathrm{I})]\right\} .
$$

The half-length $a$ will be equal to zero for any spacing smaller than

$$
h \leqslant\left[8 \pi /(\pi+\mathrm{I})^{2}\right]\left(K_{\mathrm{c}} / T\right)^{2} \approx \frac{3}{2}\left(K_{\mathrm{c}} / T\right)^{2} .
$$

\section{Estimate of error of the approximate solution}

There is one difficulty with the approximate solution given by Equations (5), (7), (I I) and (14). Although $D(y)$ is continuous throughout the range $-a \leqslant y \leqslant a$ its derivative $-B(y)$ changes discontinuously at $y= \pm h$ and at $y= \pm(a-h)$. However, the jumps in the value of $B(y)$ at the "joints" between the segments of the approximate solution can be eliminated by smoothing the value of $D(y)$ at the transition so that its derivative is continuous at $y= \pm h$ and $y= \pm(a-h)$.

Consider now how much the unsmoothed approximate solution might differ from the exact solution. Go back to Equation (2) and integrate the integral of the left-hand side of the equation by parts, keeping in mind that the Cauchy principal value of the integral is to be used. Equation (2) becomes (in the limit of $\epsilon \rightarrow 0$ )

$$
\begin{aligned}
{[\mu / 2 \pi(\mathrm{I}-\nu)]\{D(y+\epsilon) G(-\epsilon)} & -D(y-\epsilon) G(\epsilon)- \\
& \left.-\left(\int_{-a}^{y-\epsilon}+\int_{y+\epsilon}^{a}\right) D\left(y^{\prime}\right) G^{\prime}\left(y-y^{\prime}\right) \mathrm{d} y^{\prime}\right\}=-T+\rho g|y|,
\end{aligned}
$$

where $G^{\prime}\left(y-y^{\prime}\right)=\partial G\left(y-y^{\prime}\right) / \partial y=-\partial G\left(y-y^{\prime}\right) / \partial y^{\prime}$ and it should be noted that $G^{\prime}\left(y-y^{\prime}\right)=$ $G^{\prime}\left(y^{\prime}-y\right)$ and $G\left(y-y^{\prime}\right)=-G\left(y^{\prime}-y\right)$.

Equation (22) can be rewritten with negligible error as

$$
\begin{aligned}
{[\mu / 2 \pi(\mathrm{I}-\nu)]\{D(y+\epsilon) G(-\epsilon)} & -D(y-\epsilon) G(\epsilon)- \\
& \left.-\left(\int_{y-H}^{y-\epsilon}+\int_{y+\epsilon}^{y+H}\right) D\left(y^{\prime}\right) G^{\prime}\left(y-y^{\prime}\right) \mathrm{d} y^{\prime}\right\}=-T+\rho g|y|,
\end{aligned}
$$

because $G^{\prime}\left(y-y^{\prime}\right) \approx-8(\pi / h)^{2} \exp \left(-2 \pi\left|y-y^{\prime}\right| / h\right) \approx 0$ when $\left|y-y^{\prime}\right| / h$ is large. Here $H=n h$ where $n$ is a constant taken to be sufficiently large $(n \approx 3)$ to make $G^{\prime}(H)$ very small. Near the upper crack tip Equation (23) becomes (for $y$ in the range $a-H \leqslant y \leqslant a$ ) 


$$
\begin{aligned}
{[\mu / 2 \pi(\mathrm{I}-\nu)]\{D(y+\epsilon) G(-\epsilon)} & -D(y-\epsilon) G(\epsilon)- \\
& \left.-\left(\int_{a-2 H}^{y-\epsilon}+\int_{y+\epsilon}^{a}\right) D\left(y^{\prime}\right) G^{\prime}\left(y-y^{\prime}\right) \mathrm{d} y^{\prime}\right\}=-T+\rho g|y| .
\end{aligned}
$$

Equation (23) in turn can be integrated by parts to give

$$
\begin{aligned}
-[\mu / h(\mathrm{I}-\nu)][D(y+H)+ & D(y-H)]+ \\
& +[\mu / 2 \pi(\mathrm{I}-\nu)] \int_{y-H}^{y+H} B\left(y^{\prime}\right) G\left(y-y^{\prime}\right) \mathrm{d} y^{\prime}=-T+\rho g|y|,
\end{aligned}
$$

and Equation (24) to give

$$
-[\mu / h(\mathrm{I}-\nu)] D(a-2 H)+[\mu / 2 \pi(\mathrm{I}-\nu)] \int_{a-2 H}^{a} B\left(y^{\prime}\right) G\left(y-y^{\prime}\right) \mathrm{d} y^{\prime}=-T+\rho g|y| .
$$

The displacement $D(y)$ given by Equations (5) and (7) is an exact solution of Equations (23) and (25). Hence, the approximate solution for $D(y)$ is valid in the range $H \leqslant|y| \leqslant a-H$ to within an error limit of the order of $D(y) \exp (-2 n \pi)$ or smaller.

If the approximate solution were inserted into Equation (2) (or Equations (22) through (26)) the left-hand side of the equation would not equal the right-hand side except for the values of $y$ in the range $H \leqslant|y| \leqslant a-H$ in which $E(y) \approx 0$ to a high approximation. Here $E(y)$ represents the function that must be added to the right-hand side of Equation (2) in order to restore equality between the two sides. The term $E(y)$ is the stress that would have to be applied to the crack walls in order that the approximate solution becomes an exact solution (but now, of course, for a problem with a different set of conditions).

Let $\langle E\rangle$ be given by

$$
\langle E\rangle=\left(\mathrm{I} / 2 H^{\star}\right) \int_{y-H^{\star}}^{y+H^{\star}} E\left(y^{\prime}\right) \mathrm{d} y^{\prime} .
$$

If the term $\langle E\rangle$, which is the average value of $E(y)$ near $y$ over a distance $2 H^{\star}$ where $H^{\star}$ is of the order of $h / 2$, is small in magnitude compared with $-T+\rho g|y|$ the hypothetical problem for which our approximate solution is an exact solution is virtually identical to the real problem. That is, the forces that must be exerted on the crack faces to turn the approximate solution into an exact one would be so small that were these forces then removed the change of the displacement of the crack faces would be small in magnitude compared with the displacement itself.

The stress $E(y)$ is largest at the "joints" of the approximate solution where its different segments meet. To find $E(y)$ near the joint $y \approx h$ set the approximate solution into Equation (25). The stress $E(y)$ is

$$
E(y) \approx(\rho g h / 4 \pi) \log |(y-h) /(y+h)|+\rho g h,
$$

near the joint. (Smoothing the approximate solution at the joint will eliminate the logarithmically infinite value of $E(y)$ at $y=h$.) At $y=h$ :

$$
|\langle E\rangle| \approx \rho g h \text {. }
$$

The average value of $E(y)$ is small compared with $T$. Moreover, $\langle E\rangle$ is of the order of or less than the value given by Equation (29) only over a distance of at most a few units of $h$. Thus, the exact and the approximate solution of $D(y)$ must differ near the joint at $y=h$ by an amount of the order of or less than $(\rho g h / T) D(y)$. 
Now consider $E(y)$ near the joint $y=a-h$ which is close to the crack tip. Equation (26) can be rewritten to give $E(y)$ as

$$
E(y)=[\mu / 2 \pi(\mathrm{I}-v)] \int_{a-2 H}^{a} B\left(y^{\prime}\right)\left\{G\left(y-y^{\prime}\right)+(2 \pi / h)\right\} \mathrm{d} y^{\prime}+T-\rho g y .
$$

For $y>a-h$ Equation (30) becomes

$$
E(y) \approx[h / 4 \pi] \int_{a-2 H}^{a-h} \rho g\left\{G\left(y-y^{\prime}\right)+(2 \pi / h)\right\} \mathrm{d} y^{\prime},
$$

and for $y<a-h$ it becomes

$$
E(y) \approx[\mu / 2 \pi(\mathrm{I}-\nu)] \int_{a-h}^{a} B\left(y^{\prime}\right)\left\{G\left(y-y^{\prime}\right)+(2 \pi / h)\right\} \mathrm{d} y^{\prime},
$$

where $B\left(y^{\prime}\right)$ is given by the derivative of Equation (14). The average value of $E(y)$ near the joint is given by

$$
\langle E\rangle=(\mathrm{I} / h) \int_{a-3 h / 2}^{a-h / 2} E(y) \mathrm{d} y .
$$

From Equation (3I) when $a-h<y<a-h / 2$ :

$$
E(y) \approx \rho g(H-h / 2)-(\rho g h / 4 \pi)[z \operatorname{coth} z+\log |\sinh z|]_{z_{1}}^{z 2},
$$

where $z_{1}=(\pi / h)(y-a+2 H)$ and $z_{2}=(\pi / h)(y-a+h)$. From Equations (32) and (14) when $a-3 h / 2<y<a-h$ :

$$
\begin{aligned}
E(y) \approx & {[(T-\rho g a) / 4 \pi] \int_{a-h}^{a}\left\{G\left(y-y^{\prime}\right)+(2 \pi / h)\right\}\left\{2 y^{\prime}-2 a+h+(h / \pi)\right\} \times } \\
& \times\left\{\left(a-y^{\prime}\right)^{-\frac{1}{2}}\left(y^{\prime}-a+h\right)^{-\frac{1}{4}}\right\} d y^{\prime} \\
= & {[(T-\rho g a) / 2 \pi] \int_{-h^{\star}}^{h^{\star}}\left\{G\left(y^{\star}-y^{\prime \star}\right)+\left(\pi / h^{\star}\right)\right\}\left\{y^{\prime \star}+\left(h^{\star} / \pi\right)\right\}\left\{\left(h^{\star 2}-y^{\prime \star 2}\right)^{-\frac{1}{2}}\right\} d y^{\prime \star}, }
\end{aligned}
$$

where again $h^{\star}=h / 2$ and $y^{\star}=y-a+h^{\star}$ and $y^{\star}=y^{\prime}-a+h^{\star}$.

For $y<y^{\prime}$ (or $y^{\star}<y^{\star}$ ) it can be verified by graphical plotting or by numerical tabulation that the kernel $G\left(y^{\star}-y^{\prime \star}\right)$ lies between the limits

$$
-\left(\pi / h^{\star}\right)+\left(c^{\star} / h^{\star}\right)+\left(y^{\star}-y^{\prime \star}\right)^{-1}<G\left(y^{\star}-y^{\prime \star}\right)<-\left(\pi / h^{\star}\right)+\left(c^{\star} / h^{\star}\right),
$$

when $-2 h^{\star}<y^{\star}<-h^{\star}$ and $-h^{\star}<y^{\star \star}<h^{\star}$. Here $c^{\star}=0.3352$.

If Equation (36) is inserted into Equation (35) the following limits on $E(y)$ is found for $y$ in the region $a-3 h / 2<y<a-h$ (or $\left.-2 h^{\star}<y^{\star}<-h^{\star}\right)$

$$
\begin{aligned}
{[(T-\rho g a) / 2 \pi]\left\{c^{\star}+\int_{0}^{h^{\star}} y^{\star \star}\left\{\left(y^{\star}-y^{\star \star}\right)^{-1}\left(h^{\star 2}-y^{\prime \star 2}\right)^{-1}\right\} \mathrm{d} y^{\prime \star}\right\}<} \\
<E(y)<[(T-\rho g a) / 2 \pi] \times \\
\left.\times\left\{c^{\star}+\int_{-h^{\star}}^{0} y^{\prime \star}\left\{y^{\star}-y^{\prime \star}\right)^{-1}\left(h^{\star 2}-y^{\prime \star 2}\right)^{-\downarrow}\right\} \mathrm{d} y^{\prime \star}\right\} .
\end{aligned}
$$


Equation (37) reduces to

$$
\begin{gathered}
{[(T-\rho g a) / 2 \pi]\left\{c^{\star}-(\pi / 2)-y^{\star}\left(y^{\star 2}-h^{\star 2}\right)^{-\frac{1}{2}} \cos ^{-1}\left(-h^{\star} / y^{\star}\right)\right\}} \\
<E(y)<[(T-\rho g a) / 2 \pi] \times \\
\times\left\{c^{\star}-(\pi / 2)+y^{\star}\left(y^{\star 2}-h^{\star 2}\right)^{-\frac{1}{2}} \cos \left(-h^{\star} / y^{\star}\right)-\pi y^{\star}\left(y^{\star 2}-h^{\star 2}\right)^{-\frac{1}{2}}\right\} .
\end{gathered}
$$

If Equations (34) and $\left(3^{8}\right)$ are inserted into Equation (33) and use is made of the numerical evaluation of the integral $\int_{1}^{2} x\left(x^{2}-\mathrm{I}\right)^{-\frac{1}{2}} \cos ^{-1}(\mathrm{I} / x) \mathrm{d} x=\mathrm{I} . \mathrm{I} 2 \mathrm{I}$ the following limits on $\langle E\rangle$ are found:

$$
\text { -0.009 12 }(T-\rho g a)<\langle E\rangle\langle 0.245(T-\rho g a) \text {. }
$$

Using numerically calculated values of $G\left(y^{\star}-y^{\star}\right)$ it can be shown that the left-hand limit of Equation (39) can be raised to a value greater than zero. Thus

$$
0<\langle E\rangle<0.245(T-\rho g a) \text {. }
$$

Moreover, examination of numerical values of $G\left(y^{\star}-y^{\prime \star}\right)$ makes it clear that while $\langle E\rangle$ is smaller than the right-hand limit of Equation (40), the term $\langle E\rangle$ is of the order of magnitude of this limit.

The average stress $\langle E\rangle$ differs from the stress $(T-\rho g a)$ by $24.5 \%$ or less. This difference is considerably larger than that at the joint $y=h$ but it is not so large that it makes the approximate solution an unreasonable one. The implication of the positive value of $\langle E\rangle$ is that a tensile stress would have to be applied at the crack faces near the joint $y=a-h$ in order to make the approximate solution an exact one. In other words, forces that tend to close up the crack faces near the crack tips must be applied at the crack faces in order to make the approximate solution an exact one. Thus, the stress intensity factor $K$ given by Equation ( 18 ) is actually somewhat smaller than the true stress intensity factor. Suppose the value of $T$ were increased so that the value of the expression $T-\rho g a$ is increased by $24.5 \%$. The value of $K$ given by Equation (18) also is increased by $24.5 \%$. Now this increase in $K$, which is produced by increasing the tensile stress at all values of $y$, must be larger than that produced by changing the stress by $24.5 \%$ over only a limited range of $y$ of the order of $h$. Hence, the value of true stress intensity factor will lie between that given by Equation (I8) and a value $24.5 \%$ greater than it.

I believe that the equations of this section have demonstrated that our approximate solution is not an unreasonable one. They show that the approximate solution gives a conservative estimate of the stress intensity factor $K$. The actual value of $K$ is somewhat higher than the value we have calculated.

\section{Application to closely spaced, WATER-Free Grevasses}

The analysis so far has dealt with closely spaced, parallel cracks in an infinite solid. What changes would be needed in this analysis if the infinite solid is cut on the $y=0$ plane to make two infinite half-spaces with an infinite set of parallel edge cracks that penetrate to a distance $a$ from the surface? To a first approximation no change at all need be made. This claim is justified as follows. For the moment let $\sigma_{y y}(y)$ be equal to zero before the cracks were introduced into the infinite medium. Next let the set of closely spaced cracks be placed in the infinite medium. The presence of the cracks will give rise to a stress component $\sigma_{y y}(x, y)$. On the $y=0$ plane the value of $\sigma_{y y}$ averaged over a distance $h$ in the $x$ direction must equal zero. (If it were not equal to zero there would be a net vertical force on a block of material bounded by the planes $y=0$ and $y=y_{0}$, where $y_{0}$ is a distance very large compared with the length $a$.) Now the crack faces are traction-free and the cracks extend in the $y$ direction for a distance large compared with $h$. Hence, it is unlikely for the derivative $\partial \sigma_{y y} / \partial x$ to have a 
large magnitude on the $y=0$ plane. The maximum magnitude of $\sigma_{y y}$ on the $y=0$ plane is of the order of $(h / a) T$. (Estimated from the stress fields of the collection of infinitesimal dislocations that would produce the crack opening displacement $D(y)$.) Thus, as a first approximation $\sigma_{y y}(x, 0)$ as well as the average value of $\sigma_{y y}(x, 0)$ can be taken equal to zero. As a result the infinite medium can be cut along the $y=0$ plane without altering the solution. (By a symmetry argument the shear stress $\sigma_{x y}$ is equal to zero across the $y=0$ plane.)

In a solid in the Earth's gravitational field, a body force $\rho g$ acts on any unit volume element. In a glacier this body force sets up the hydrostatic stress components $\sigma_{y y}=\sigma_{x x}=$ $-\rho g y$, where $y$ is distance measured downwards from the upper surface of the glacier. The stress component $\sigma_{y y}$ cannot alter the crack solution just found. The crack planes are normal to the planes across which the stress component $\sigma_{y y}$ acts. Thus the stress component $\sigma_{y y}$ can set up no surface traction on the crack faces.

If, in addition to the hydrostatic stress, a tensile stress $\sigma_{x x}=T$ exists in a glacier, crevasses can be opened up in the glacier. The profile of these crevasses, if they are closely spaced, are given by Equations (5), (I I) and (I4). If they are widely spaced their profiles will be given by equations derived in my older paper (Weertman, I973).

\section{Conclusion}

The profile of a crevasse which is one of a set of closely spaced, water-free crevasses in a glacier that is subjected to a tensile stress $T$ in addition to the hydrostatic pressure is given by Equations (5), (I I) and (I 4 ) of the text. If the fracture strength of ice is essentially zero the depth of penetration of the crevasses is equal to $T / \rho g$, the depth first deduced by Nye. The depth is independent of the spacing between the crevasses. This conclusion is in disagreement with the results of the analysis by $\mathrm{R}$. A. Smith. If the fracture strength of ice is finite the depth of penetration is given by Equation (20) and it does depend on the crevasse spacing. No penetration occurs if the spacing is smaller than the value ${ }_{2}^{3}\left(K_{\mathrm{c}} / T\right)^{2}$, where $K_{\mathrm{c}}$ is the fracture toughness of ice. For a perfectly brittle solid the value of $K_{\mathrm{c}}=\left\{2 E_{\gamma} /\left(\mathrm{I}-\nu^{2}\right)\right\}^{\frac{1}{2}}$ where $E$ is Young's modulus and $\gamma$ is the surface energy of the solid. If ice is considered to be a perfectly brittle solid the value of $K_{\mathrm{c}}$ is approximately $45 \mathrm{kN} / \mathrm{m}^{\frac{3}{2}}$ (using $E=9 \mathrm{GN} / \mathrm{m}^{2}$ and $\gamma=0.1 \mathrm{I} \mathrm{J} / \mathrm{m}^{2}$ ). For $T=0.1 \mathrm{MN} / \mathrm{m}^{2}$ ( $\mathrm{I}$ bar) no crevasses will form for spacings smaller than $0.3 \mathrm{~m}$.

The results of the analysis of this paper are not restricted to the problem of closely spaced crevasses in glaciers. They can also describe the behavior of cracks at the surface of the Earth. A practical application of the analysis exists in the field of geothermal energy. To extract heat from hot, dry rock masses it is necessary to fracture the rock hydraulically and let fluid flow through the large cracks that are created. It is hoped that secondary cracks will form spontaneously on the surfaces of these large cracks because of the large tensile thermal stresses that are set up when the flowing fluid cools the rock. The (tensile) thermal stresses decay with distance from the crack faces in an analogous manner to that by which the stress in a glacier can change from tensile at the upper surface to compressive at depth. (The secondary cracks are very desirable because they increase the transfer efficiency of heat from the rock to the fluid.) Our equations set limits on the spacing between the secondary cracks.

\section{Acknowledgements}

This work was supported by the National Science Foundation under Grant No. AER 75 -00 I 87 .

MS. received 13 January 1976 and in revised form 7 June 1976 


\section{REFERENCES}

Ichikawa, M., and others. 1965. Interaction between parallel cracks in an elastic solid and its effect on fracture, [by] M. Ichikawa, M. Ohashi and T. Yokobori. Reports of the Research Institute for Strength and Fracture of Materials, Tohoku University, Vol. I, No. I, p. I-14.

Nye, J. F. 1955. Comments on Dr. Loewe's letter and notes on crevasses. Fournal of Glaciology, Vol. 2, No. 17 , p. 512-14. [Letter.]

Robin, G. de Q. 1974. Depth of water-filled crevasses that are closely spaced. Fournal of Glaciology, Vol. 13, No. 69 , p. 543. [Letter.]

Smith, E. I966[a]. The interaction between dislocation-type cracks. III. Infinite periodic sequences of noncoplanar cracks. Proceedings of the Royal Society of London, Ser. A, Vol. 295, No. 1443, p. 429-39.

Smith, E. 1966[b]. The opening of parallel cracks by an applied tensile stress. International fournal of Engineering Science, Vol. 4, No. 1, p. 41-52.

Smith, R. A. 1976. The application of fracture mechanics to the problem of crevasse penetration. Fournal of Glaciology, Vol. 17, No. 76, p. 223-28.

Weertman, J. 1971. Theory of water-filled crevasses in glaciers applied to vertical magma transport beneath oceanic ridges. Journal of Geophysical Research, Vol. 76, No. 5, p. $1171-83$.

Weertman, J. 1973. Can a water-filled crevasse reach the bottom surface of a glacier? Union Géodésique et Géophysique Internationale. Association Internationale d'Hydrologie Scientifique. Commission de Neiges et Glaces. Symposium on the Hydrology of Glaciers, Cambridge, 7-13 September 1969, p. 139-45. (Publication No. 95 de l'Association Internationale d'Hydrologie Scientifique.)

Weertman, J. 1974. Depth of water-filled crevasses that are closely spaced. Fournal of Glaciology, Vol. I3, No. 69, p. 544. [Letter.]

Yokobori, T., and Ichikawa, M. ${ }_{1967}[\mathrm{a}]$. The interaction of parallel elastic cracks and parallel slip bands respectively based on the concept of continuous distribution of dislocations. I. Parallel slip bands and their stress concentration. Reports of the Research Institute for Strength and Fracture of Materials, Tohoku University, Vol. 3, No. 1, p. 1-14.

Yokobori, T., and Ichikawa, M. ${ }_{1967}[\mathrm{~b}]$. The interaction of parallel elastic cracks and parallel slip bands respectively based on the concept of continuous distribution of dislocations. II. Parallel elastic cracks and their effect on brittle fracture strength. Reports of the Research Institute for Strength and Fracture of Materials, Tohoku University, Vol. 3, No. 1, p. 15-37. 